Comunicación a la intemperie: en busca de un campo para pensar los desafíos contemporáneos

Javier Ares Yebra

Question, Vol. 1, N. 62, e151, abril-junio 2019

ISSN 1669-6581 | https://doi.org/10.24215/16696581e151

http://perio.unlp.edu.ar/ojs/index.php/question

FPyCS | Universidad Nacional de La Plata

La Plata | Buenos Aires | Argentina

\title{
Comunicación a la intemperie: en busca de un campo para pensar los desafíos contemporáneos
}

\author{
Communication out in the open: looking for a field to think \\ contemporary challenges
}

\author{
Javier Ares Yebra javieraresyebra@gmail.com \\ https://orcid.org/0000-0002-1027-7762 \\ Universidad de Vigo (España)
}

Academia Argentina de Artes y Ciencias de la Comunicación (Argentina)

\section{Resumen}

De los escenarios de la vida cotidiana a los espacios discursivos, prácticamente no hay ámbito o disciplina -filosofía, arte, ciencia- que no aborde hoy, de algún modo, la idea de comunicación. Pero, ¿existe algo así como "la comunicación"? Considerando su importancia 
como categoría fundamental de la vida en común, el presente ensayo reflexiona sobre la comunicación como espacio de pensamiento desde el que analizar la nueva complejidad social y cultural. En segundo lugar, a través de una revisión histórica y epistemológica se problematizan las nociones de campo y objeto, examinando la idea de comunicación en otros ámbitos de conocimiento, como el filosófico, realizando desde el mismo algunas aportaciones. Por último, se sugieren algunos conceptos, como el desdoblamiento y la concentración, para tratar de cartografiar nuevos senderos en el estudio de los procesos de comunicación que den cuenta de su papel decisivo en la construcción de lugares comunes.

Palabras clave: Comunicación; objeto; campo; desdoblamiento; concentración.

\section{Abstract}

From the scenarios of everyday life to discursive spaces, there is practically no field or discipline -philosophy, art, science- that does not address today, in some way, the idea of communication. But, is there something like "communication"? Considering its importance as a fundamental category of life in common, this essay reflects on communication as a space of thought from which to analyze the new social and cultural complexity. Secondly, through a historical and epistemological review the notions of field and object are problematized, examining the idea of communication in other areas of knowledge, such as the philosophical one, making some contributions from it. Finally, some concepts are suggested, such as unfolding and concentration, to try to map new paths in the study of communication processes that account its decisive role in the construction of common places.

Keywords: Communication; object; field; unfolding; concentration.

"El hombre es nostalgia y búsqueda de comunión"

(Paz, 2015)

Las páginas que siguen prolongan algunas reflexiones suscitadas en el marco de una reciente tesis doctoral dedicada al filósofo ruso Pierre Souvtchinsky (Ares Yebra, 2017). A partir del análisis de sus fuentes musicales, las más significativas dedicadas a Igor Stravinsky, la investigación establece conexiones entre su concepción del tiempo musical, la presencia de un mecanismo perceptivo de desdoblamiento y concentración en sus escritos y la música del 
compositor ruso como objeto de reflexión. Propone además un acercamiento al estudio de la música y la comunicación ahondando, entre otras, en la idea de una comunicabilidad universal necesaria en la música, atendiendo al pensamiento musical no como un pensamiento de la no comunicación, sino como un pensamiento de la comunicación sin concepto (Lyotard, 1998).

Durante la investigación desarrollé un particular interés en trabajar posibles relaciones entre los procesos de percepción, el estudio de los fenómenos culturales y el modo en que éstos toman forma en términos de experiencias temporales. Asimismo, se reveló la importancia de la narratividad de la historia como articulación de la experiencia de una época, entendiendo, como señalara Boris Pasternak, que "la historia es significativa porque es simbólica" (1959: 282).

Más tarde, ya inmerso en nuevas lecturas para la preparación de este texto, observé que ambas cuestiones, la producción y percepción de nuevas experiencias de tiempo -desde los procesos de intercambio hasta las nuevas pautas de consumo cultural-, y la historia como cronotopo, representan para Lawrence Grossberg "dos de los modos más comunes en que se ha entendido la modernidad" (2012: 12). Tiempo y narración pueden constituir dos claves para pensar la Modernidad, y en definitiva cualquier época, ya que, como sugiere Ernesto Laclau, "todo tiempo se da una imagen de sí mismo, un cierto horizonte -tan borroso e impreciso como se quiera- que unifica en cierta medida el conjunto de su experiencia" (2000: 19).

Si algo reveló la tesis en lo que a investigación en comunicación se refiere, es el déficit de certezas, la multitud de interrogantes, la falta de consenso y la dispersión (Budd y Ruben, 1972; Anderson, 1996). ¿Existe una (inter/multi/in/trans) disciplina-campo-ciencia de la comunicación? ¿Se trata, más bien, de una categoría filosófica o, como afirma Martín-Barbero, de una "dimensión constitutiva de la cultura y por tanto de la producción de la sociedad" (1992: 1)? ¿Y si debemos abordarla como una competencia cognitiva, una certeza antropológica? Para terminar de complicarlo, ¿y si hubiese algo de cierto en todo lo anterior? En caso de reconocer la existencia de un estatuto disciplinario de la comunicación, el diagrama de inquietudes se abriría en otra dirección. ¿Cuál es su origen y su identidad como campo? ¿De qué forma generar conocimiento sobre algo que usamos constantemente? ¿Habría que empezar por plantear algo así como las formas de la comunicación y la historia? ¿Qué particularidades tiene la investigación en comunicación? $\mathrm{Y}$ entre estas particularidades, ¿qué supone la interdisciplinariedad en su estudio? ¿Por qué no parece posible cerrar todo este debate?

Son numerosos los esfuerzos por tratar de responder a estas preguntas. A pesar de ello, permanece una gran zona de sombra en torno a la dialéctica objeto-campo. En la mayoría de los estudios se advierte un fuerte cuestionamiento de la comunicación como objeto y de su configuración como campo de conocimiento. La definición y la identidad se presentan como los 
dos grandes desafíos de la comunicación. Sin embargo, pocas investigaciones tratan de superar el debate endogámico y de proyectar sus reflexiones sobre la comunicación hacia un ámbito de mayores dimensiones. ¿Desde dónde y en qué dirección enfocar hoy su estudio? ¿Cómo conjugar estas preocupaciones con los desafíos propios de nuestra época? Hacer frente a estos desafíos -al destino, como escribiera Bataille-, ha de ser, precisamente, lo esencial del conocimiento.

Responder a las preguntas planteadas es una tarea compleja que, sin embargo, se presenta como inevitable. Desde esa necesidad de abordarlas, este ensayo se plantea varias hipótesis como ejes de trabajo. En primer lugar, es posible trabajar en una misma propuesta con dos afirmaciones aparentemente contrapuestas. Por un lado, la comunicación constituye una categoría fundamental de nuestra época. Por otro, siempre ha existido una idea de comunicación, en cuyo caso se volvería totalmente pertinente una revisión histórica. La combinación de dos hipótesis aparentemente contrarias -sincrónica y diacrónica- ha sido sugerida en el ámbito de la investigación en comunicación por autores como, por ejemplo, Jesús Galindo (2008).

En línea con lo que plantea Pablo Rieznik (2007) en sus investigaciones sobre la idea de trabajo, es esencial distinguir desde un principio un sentido histórico y un sentido antropológico de la idea de comunicación. El primero hace referencia a las formas que ha adoptado la comunicación a lo largo de la historia. El segundo alude a la comunicación como condición humana. Abordar el concepto de comunicación requiere la combinación de una perspectiva antropológica con una aproximación histórica. Es posible, además, plantear el estudio de la comunicación como desafío a la realidad social, y no simplemente como una descripción o abstracción de ésta, tratando de integrarla a un proyecto general de carácter humanista.

\section{Actualidad de la comunicación}

"Hoy, ni escena ni espejo, sino pantalla y red" (Baudrillard, 1997)

Pensamos inevitablemente desde la experiencia de nuestra época. Sin embargo, se advierte un giro decisivo, radical, en relación con la percepción del tiempo. Vivimos la ausencia de una experiencia de la duración, un tiempo acelerado, que afecta pero que no que significa (Han, 2015), inmersos en una sociedad de lo provisorio que asiste a la fragmentación de uno de sus principios fundamentales: la crisis de la modernidad es la crisis de la razón como experiencia 
de un centro. La pérdida de esta "fortaleza inexpugnable" (Jarauta, 1991) da origen a múltiples discursos, que se entrelazan tratando de afrontar innumerables desafíos de tipo identitario, cultural, social y político.

En este contexto resulta muy complejo proponer herramientas para poder interpretar la realidad. Con independencia del punto de partida, la perspectiva o el campo de conocimiento, las dificultades, en forma de "condiciones externas" (Feyerabend, 1986) no permiten al investigador ser demasiado estricto en la construcción de su mundo conceptual. El estado de cosas cambia constantemente. Es antes que nada este clima de época el que nos interpela para proseguir con la necesaria tarea teórico-ensayística.

Al mismo tiempo que aumenta la literatura preocupada por esclarecer el conflicto de la cultura contemporánea, se multiplican a un ritmo no menor los debates marcados por ese éxtasis de la comunicación (Baudrillard, 1997) que vive la sociedad actual. La (hiper)comunicación inunda no solo los espacios cotidianos, sino también los discursivos, y ya es habitual encontrarla formando parte de las investigaciones artísticas y filosóficas. En cuanto a actividad de producción de conocimiento se refiere, estamos inmersos en una coyuntura histórica en la que proliferan las teorías, las aproximaciones y los modelos comunicacionales, sin que por ello hayamos llegado a un consenso sobre cuál es el objeto de los llamados estudios en comunicación. Por más que la saturación alimente la ilusión de un campo disciplinar, el debate no se da en plenas condiciones porque, precisamente, la discusión no cuenta con el mínimo de acuerdo necesario.

La lectura histórica - ¿el primer problema es el estado de la cuestión?- determina los hechos y conceptos fundamentales de un campo de conocimiento. De este modo, atendiendo a nuestra interpretación de la idea de comunicación, podemos considerar, por ejemplo, la prensa, la radio, la televisión e internet como medios, pero también el coro en la tragedia griega. La perspectiva histórica tiene, por tanto, consecuencias inmediatas. Varía el relato clásico, el discurso hegemónico. Es por ello que al cartografiar la idea de comunicación emergen determinadas problemáticas que nos complican pactar previamente textos y hechos de fundación, porque intuimos que hay otra forma de contar la historia del pensamiento comunicacional, actuar con otra lógica narrativa para ampliar así el panorama conceptual y las formas en las que construimos conocimiento.

Observando el entorno cotidiano, un trayecto en transporte público o un paseo por la avenida de cualquier ciudad, nos percatamos de que comunicar(se) representa hoy la principal acción de una práctica global. Los procesos sociales de intercambio se configuran sobre flujos de información cada vez más acelerados, instantáneos, sin apenas margen para la interpretación crítica. Constantemente somos interpelados por una nueva publicidad o el sonido del siguiente 
whatsapp. Volviendo al uso de pictogramas, nuestros mensajes se condensan, ganan cierta rapidez de transmisión, pero a cambio, nuestra comunicación no dura. $\mathrm{O}$, más bien, se establece una nueva sintaxis fragmentada de la duración, del intercambio, basada, justamente, en la discontinuidad.

"Hoy, ni escena ni espejo, sino pantalla y red" (Baudrillard, 1997: 9). El individuo, que ha perdido la significación dada por el sistema de los objetos - ¿ha desaparecido la oposición sujeto/objeto tal y como la conocíamos?-, se convierte en una "terminal de múltiples redes". Nos hemos aislado de lo que nos rodea, de esa realidad significativa al alcance de la mano. Si un prefijo sintetiza nuestra época es el de tele -teléfono, televisión-, lo cual es suficientemente demostrativo de la distancia que hemos interpuesto entre nosotros y el mundo. La comunicación se ha convertido en un elemento totalizador de la vida cotidiana. Las nuevas tecnologías, extensiones (McLuhan, 1964) del pensamiento, proporcionan toda suerte de soportes digitales en una espacialización sin precedentes de la interioridad que termina por evaporar nuestra dimensión psicológica. De este modo, han variado el paradigma clásicocartesiano de la filosofía que igualaba el ser al pensar. Ser es comunicarse. La introspección ha dejado paso a una configuración de reciprocidades en la que la construcción de identidades apunta casi con exclusividad al ser como ser en red.

\section{Dos problemas: definición e identidad de la comunicación}

"Una investigación empieza con un problema"

(Karl Popper, 2008)

El ámbito de la investigación en comunicación afronta, dos cuestiones pendientes, dos desafíos. El primero tiene que ver con su objeto de estudio: el problema de la definición de la comunicación. El segundo, el problema de su identidad, atiende a su constitución como campo, sus límites y competencias disciplinares. La revisión histórica de los estudios en comunicación pasa por considerar en paralelo ambas cuestiones, objeto y campo, definición e identidad. Esta construcción del relato histórico de la comunicación es al mismo tiempo una revisión epistemológica. 


\section{La comunicación como objeto o el problema de la definición}

El espacio de la comunicación es hoy un lugar estratégico de pensamiento. En este sentido, para poder constituir ese lugar, Martín-Barbero propone "perder la obsesión por el objeto propio para empezar a observar en serio los procesos reales en los que se produce la comunicación [...] llegar a la teoría pero desde los procesos de comunicación, desde la opacidad". Y añade que "debemos de empezar a pensar los procesos de comunicación no desde las disciplinas, sino desde los problemas y las operaciones del intercambio social [...] desde las matrices de identidad y los conflictos que articula la cultura" (2012: 76).

La cantidad de recortes posibles al laberinto de interacciones que suponen los procesos cotidianos de intercambio social, el crecimiento exponencial de literatura especializada, y el propio carácter polisémico del concepto, complejizan el consenso en torno a cualquier definición de la comunicación. Siendo múltiples las aproximaciones, citaré únicamente algunas de ellas para exponer esa complejidad. En su libro Las funciones de la Comunicación Humana: una aproximación teórica, Frank Dance y Carl Larson realizan una lista con 26 definiciones del término comunicación (1976). Reflexionando sobre las dificultades de plantear una definición, Theodore Clevenger afirma que:

El continuo problema sobre la definición de la comunicación por escolares o propuestas científicas proviene del hecho de que el verbo «comunicar» está bien establecido en el léxico común y por tanto no es sencillo capturarlo para su uso científico. De hecho, es uno de los términos más utilizados en inglés (1) (Littlejohn y Foss, 2005: 12).

Por su parte, Jesús Galindo (2008) distingue cuatro significados-acciones del término: transmitir (difusión), conectar (estructuración), intercambiar (interacción) y efecto de comunión (acción a partir de lo compartido). Cada una parece apuntar a diferentes conceptos centrales, en una especie de juego de pares dialécticos. La idea de comunicación como difusión, por ejemplo, a los medios. La estructuración a la conectividad. La interacción parece referirse a una comunicación de procesos, y el énfasis en la comunión a la expresión. El mundo-red en el que vivimos se configura a partir de la idea de comunicación como conectividad. En este sentido, Parag Khanna (2017) defiende que esta conectividad es la mayor fuerza transformadora del siglo XX, base de la que denomina como geografía funcional o conectografía, en una lectura de la comunicación en la que prevalece todo lo que tiene que ver con infraestructuras. La articulación entre conectividad e infraestructuras propuesta por Khanna está presente también en los textos del Comité Invisible (2014: 49-58), en los que esta relación es la base de una lectura logística del poder. 
El hombre tiende a convertir en objetos dimensiones esenciales de sí mismo, a transformar atributos antropológicos en características del mundo de las cosas. A su vez, se manifiesta en él una poderosa inclinación, arraigada en su naturaleza social, a proyectar características propias de los objetos físicos a los hechos psíquicos. A la pregunta por el significado del término comunicación, no deberíamos de haber respondido convirtiéndola en un objeto. Tendría que haber seguido siendo principalmente un atributo, y no una sustancia. Siguiendo la misma idea que propone el Comité Invisible (2017: 66) para abordar la idea de política, se puede sugerir que no hay algo así como "la comunicación": es comunicacional todo lo que guarda relación con el encuentro, la interacción y el intercambio entre formas de vida, entre sensibilidades. La comunicación sucede inmediatamente con la vida, nace de lo próximo, lo concreto, lo cotidiano. Forma parte de la experiencia interior y, al mismo tiempo, constituye un aspecto esencial de la sociedad. El pensamiento contiene y exige una condición universal de comunicabilidad, y al mismo tiempo la comunicación es en sí misma un hecho exterior, algo que se hace, una extensión de ese pensamiento en el mundo.

Retrotraer el estudio de la comunicación al origen del ser y de la vida genera el riesgo de trasladar su investigación al terreno de la disertación filosófica, o a una especie de antropología de las formas y de los procesos simbólicos y culturales. Sin embargo, puesto que lo atraviesa todo, es necesario atender a su presencia en todos los ámbitos, lo cual, por otro lado, constituye un ejercicio de una utilidad y una riqueza incuestionables. En este sentido, Karl Jaspers vincula precisamente la comunicación al estudio del ser y del lenguaje:

[...] la comunidad de todos los hombres no puede basarse en el culto universal de una verdad una y única, sino tan solo en el medio de comunicación compartido por todos. Desarrollar la conciencia de este medio...es la tarea de la lógica filosófica, la que determina por un lado, las condiciones que deben darse para que se opere la incondicional voluntad de comunicación, y por el otro, las modalidades de ruptura de la comunicación, su sentido y sus consecuencias (Jaspers, 1964: 84).

\section{La comunicación como campo o el problema de la identidad}

Al conquistar la inmediatez comunicativa a través de las nuevas tecnologías -ese permanente "estar ahí" conectados-, la dimensión espacial de la idea de campo se ha comprimido. Incluso la dimensión física del término ha quedado, en cierto modo, obsoleta. En palabras de Baudrillard, "el inmenso campo geográfico parece un cuerpo desértico cuya extensión resulta innecesaria (y que aburre atravesar, incluso al margen de las autopistas)" (1997: 14). Al mismo tiempo, las nuevas formas de comunicación ha nminiaturizado los entornos en los que se Question, Vol. 1, N. 62 , abril-junio 2019. ISSN 1669-6581 
desarrollan los procesos de comunicación. Ya es mayoritario el tipo de intercambio entre sujetos que no están presentes uno frente al otro. Esta desaparición de la presencia, esta "pérdida del paisaje como escena" señalada por Baudrillard, es también la desaparición del espacio público. A medida que este desaparece, la publicidad lo invade todo. En este contexto, plantear un campo propio para la comunicación es legítimo, sin duda, pero resulta realmente complejo. ¿Responde, más bien, a la obsesión positivista por fraccionar el conocimiento en dominios específicos? En lugar de compartimentos estancos o campos delimitados, podemos ver el conocimiento como un océano en constante crecimiento, en el que "toda teoría particular, todo cuento de hadas, todo mito, forman parte del conjunto que obliga al resto a una articulación mucho mayor" (Feyerabend, 1986: 14).

Una breve revisión de la literatura revela ostensibles diferencias en el tratamiento y la importancia que le dan los investigadores al problema de la identidad de la comunicación. Luis Ricardo Sandoval se refiere a ella como una "actividad de producción de conocimiento científico" y señala que "la pertenencia al campo supone formas específicas de producción" (2015: 16). Para Gustavo León Duarte "Ios desafíos que en la actualidad se le presentan a su investigación, tienen que ver fundamentalmente con su estatuto disciplinario" (2010: 70).

Son numerosos los trabajos que abordan este debate concentrándose en torno a la consolidación académica de los estudios en comunicación. Precisamente, factores como su gran crecimiento académico en los últimos años han derivado, según Wolfgang Donsbach (2006) en una pérdida de identidad del campo. Por su parte, Marta Rizo afirma que "la comunicación no se ha configurado ni como ciencia ni como disciplina. Pero no hay duda de que la institucionalización y la cultura académica que existe sobre el fenómeno comunicativo [...] permiten hablar de un campo académico" (2012: 26). Para Sandoval (2015), Rizo interpreta la denominación de campo como un déficit, como la opción de descarte una vez que el cuerpo de conocimientos en cuestión no llega a calificar como ciencia o disciplina.

Otra cuestión a revisar en el ámbito académico es la expresión, ya normalizada, de "ciencias de la comunicación", empleada en la enseñanza universitaria (al menos en España) para designar a las carreras de comunicación, periodismo y publicidad, derivada a su vez de la forma "ciencias de la información", y cuya demanda ha crecido de manera exponencial en los últimos años. Esta expresión no es denotativa de ningún tipo de consideración científica sobre un campo sólido y determinado del saber, sino que alude únicamente a un conjunto de disciplinas que, supuestamente, se ocupan de un mismo objeto, la comunicación. Poco nos aporta a la doble problemática de la definición y de la identidad del campo la comprobación de que varias disciplinas atraviesan un mismo objeto de estudio. 
Numerosas investigaciones optan por trasladar el foco de la comunicación como campo académico a la comunicación como concepto (inter-trans-multi)disciplinar. Estas referencias al carácter "interdisciplinar" (Craig, 1999), "posdisciplinar" (Barberis, 2015), "transdisciplinar" (Martín-Barbero, 1992, 2007; Vidales Gonzáles, 2017), e "indisciplinar" (Andacht, 2001) de la comunicación son hoy habituales, pero bien podrían acompañar a cualquiera de las ciencias sociales, por lo que nada o casi nada parecen aportar a su estudio (2). Además, si lo que se pretende es la constitución de un campo específico, siguiendo esa obstinación positivista por acotar un lugar propio para la comunicación, la alusión permanente a la presencia de otras disciplinas no parece el modo más útil de constituir una propia. Sin duda la riqueza teórica es un síntoma de fortaleza. Sin embargo, lejos de un enriquecimiento, la mención a la interdisciplinariedad indica únicamente un acercamiento múltiple a lo mismo, no nos informa sobre la doble problemática propuesta-definición e identidad- ni nos dice realmente mucho de cara a poder saber si la comunicación constituye un saber particular sin dependencia explícita de otras disciplinas. En definitiva, debido a su uso sistemático, estas adjetivaciones están más cerca de la redundancia o la confusión que de aportar algún nuevo matiz, una nueva perspectiva sobre el fenómeno comunicativo. ¿Qué ciencia social no sería interdisciplinar si, justamente, todas se ocupan de lo mismo y con procedimientos tan similares?

La comunicación surge de la necesidad de encuentro, de intercambio, de comunión. Como práctica social, acudimos a ella constantemente. Es justamente la sedimentación tan brutal de esta experiencia cotidiana la que genera dudas acerca de la legitimidad de la comunicación como campo. Un campo en el que, como señala Mattelart, "[...] pensar que se puede hacer tabla rasa de esta sedimentación y que en esta disciplina, a diferencia de otras, todo está por crear es poderoso" (1997: 13).

\section{Aspectos históricos y perceptivos de la comunicación}

El tratamiento de la comunicación en el tiempo plantea inevitablemente la cuestión del posible origen del campo comunicacional. Desde los primeros intentos de constituir un ámbito específico de estudio, la comunicación presenta cierta dualidad que la caracteriza no solo desde el punto de vista de su percepción como objeto y como campo, sino también desde una revisión histórico-epistemológica. 


\section{Notas sobre el carácter dual de la comunicación. Una aproximación histórica}

Dos acepciones dominan históricamente la lectura del concepto de comunicación: transmisión y ritual. Así las encontramos, por ejemplo, en Comunicación como Cultura, de James Carey (1992). Antes que él, John Dewey ya había señalado que la comunicación tiene dos definiciones contrastantes en la historia del pensamiento occidental, y usó el conflicto entre ambas como una fuente de tensión creativa en su trabajo. En su aproximación histórica, Cisneros también sugiere una dualidad en torno al concepto:

Históricamente se han dado cuando menos estos dos sentidos al concepto de comunicación: como persuasión y como entendimiento. Ambos con implicaciones muy divergentes tanto en el análisis de los fenómenos como en el manejo de procesos y prácticas comunicacionales (Cisneros, 2002: 51).

Combinando esta aproximación con las teorías de Habermas (1999) -teoría de la acción comunicativa - y Antonio Pasquali (1990) -comunicación como interacción, descubrimiento de la presencia del otro-, Cisneros propone la idea de entendimiento como alternativa semántica. Con todo, el elemento persuasivo al que alude se sitúa en la línea de la comunicación como transmisión señalada por Carey. Por su parte, Lucien Sfez (2007) compone variaciones en torno a la idea de comunicación a partir de las dos definiciones históricas: los conceptos de representación y expresión en lugar de las nociones mencionadas de transmisión y ritual. Al mismo tiempo, Sfez propone la confusión como tercera vía.

Desdoblamiento y concentración en el estudio de los procesos de comunicación. Una propuesta

La percepción no es un elemento de la comunicación sino, más bien, una condición para que esta se produzca. Pero observarla desde ese ámbito -la comunicación como una competencia cognitiva - nos da la posibilidad de adoptar un punto de vista determinado por las realidades esenciales espacio y tiempo. En los estudios se suele obviar o pasar por alto la importancia de esta cuestión. Si nociones tan inmediatas como las de tiempo y espacio son decisivas en el desarrollo de los principios psicológicos, y constituyen el anclaje histórico de cualquier fenómeno, ¿cómo no considerarlas en relación con la comunicación? En este sentido, partiendo de la detección de un mecanismo perceptivo de desdoblamiento y concentración en los escritos de Pierre Souvtchinsky propongo la aplicación de este mecanismo para el estudio de los procesos de comunicación. Según señala el filósofo ruso: 
El término de "desdoblamiento" [...] querría indicar y precisar un fenómeno de percepción, una forma de concentración interior, donde lo sensible y lo inteligible se confunden, cuando son simultáneamente atraídos por (al menos) dos polos de atracción, cuando "ser en sí mismo"- se confunde con "ser en otra parte", "ser con otros" [...] (Souvtchinsky, 1962: 7).

El desdoblamiento se presenta como "parte de un descubrimiento, de una evidencia de tipo antropológico" (Souvtchinsky, 1982: 15-16). Puesto que este mecanismo afecta a la percepción de cualquier fenómeno en tanto experiencia temporal y también necesariamente al sujeto, que pasa a poder percibirse en varios planos temporales, propongo explorar la posible utilización de este concepto y de su par, la concentración, para estudiar los procesos de intercambio de roles que atraviesa el sujeto en las situaciones de comunicación.

En su Principios de psicología, William James sugiere también la idea de un desdoblamiento del sujeto:

Cada uno de nosotros hace una escisión de todo el universo en mitades; y para cada uno de nosotros casi todo el interés se atribuye a una de las mitades; pero todos trazamos la línea divisoria entre ellas y en un lugar diferente. Cuando diga yo que todos nosotros llamamos a las mitades con los mismos nombres, y que esos nombres son "yo" y "no yo", respectivamente, se verá de inmediato lo que quiero decir...Cada uno de nosotros dicotomiza el Cosmos en un lugar diferente (1994: 231-232).

Para comprender mejor el desdoblamiento que se produce en los procesos de comunicación en un sentido de cambio de roles, es esclarecedor el siguiente pasaje correspondiente a una conferencia de Lyotard:

Hablar es comunicar. Pero esta expresión comporta ya en potencia[...]que la comunicación sería la operación que garantiza la transmisión de un mensaje preparado a uno de los polos del sistema. Expresar sería sacar al exterior lo que permanecía en el interior [...]. No hay nada de eso. Nuestra experiencia de una palabra viva no es la de la recitación de un discurso prefabricado. Es la de una puesta a punto ante el interlocutor, ante las preguntas que nos dirige y que nos obliga a dirigir hacia lo que pensábamos, hacia nuestro propio mensaje, o lo que creíamos que lo era. Es la experiencia de un juego, es decir, de un intercambio, de una circulación de signos, y para que este intercambio no caiga en la pura y simple repetición...la comunicación implica también el intercambio de roles, implica que yo no sea simplemente yo mismo... sino también el otro, e implica del mismo modo que el otro sea también yo, es decir que el otro sea el otro de sí mismo, el distinto de sí mismo.

Question, Vol. 1, N. o 62, abril-junio 2019. ISSN 1669-6581 
Más adelante, añade:

Nunca se puede entender cómo es posible la comunicación si se comienza por encerrar cada mensaje, el del otro y el mío, en nuestras subjetividades respectivas...hablar es pensar -es inmediatamente comunicación- es decir lleva en sí la capacidad de estar al otro lado del yo [...] (Lyotard, 1989: 128-131).

La comunicación es una poética, se crea accediendo al juego de roles, al intercambio. Configura la experiencia de un juego compartido por dos o más individuos que recuerda aquella idea del hombre sobre la que Johan Huizinga (2001) fundamenta su homo ludens, y que Han (2014) prolonga en el homo digitalis. Este intercambio nos permite descubrirnos como otro, permitiendo a su vez al otro descubrir al otro de sí mismo. En este sentido, podemos afirmar que en los procesos de comunicación se produce un desdoblamiento ontológico. La comunicación tiene que ver con la ontología porque atiende inmediatamente al ser-con-losotros. Pero es también una condición general indispensable de interacción no sólo a nivel social, entre los hombres sino, en un sentido material, entre el hombre y la naturaleza. Esta actividad impuesta por la naturaleza a la vida humana es independiente de las formas que adopte la organización de la vida social y de los procesos y contextos de interacción o, mejor aún, es común a todos ellos.

Las revisiones (epistemológica, histórica, conceptual) y los intentos de constituir una disciplina institucionalizada, de cartografiar un campo académico o de articular teorías y modelos, responden a un enorme esfuerzo colectivo de integración que informa de un movimiento de concentración en torno a la comunicación. ¿Debe adquirir este movimiento necesariamente la forma de una metateoría, de un metadiscurso, como propone Craig? Enfatizando de nuevo la dificultad, ¿cómo plantear una forma concentradora de algo que usamos constantemente? Los procesos de comunicación son procesos de (re)conocimiento. En este sentido, la comunicación otorga identidades. Constituye una característica fundamental de los procesos en los que hunde sus raíces lo vivo. Informarnos de nosotros mismos y poner en común ese estar juntos, hacer comunidad.

\section{A modo de conclusión}

“¿Por qué nunca se inventó un dios de la lentitud?”

(Peter Handke, 1995) 
El falso progreso del proyecto positivista ha traído consigo una inusitada velocidad en el desarrollo de la ciencia, de los avances tecnológicos, de las relaciones sociales. En definitiva, una aceleración de la vida diaria. Pero nos hemos olvidado de cimentar ese supuesto desarrollo sobre una base sostenible, llegando a un punto en el que los problemas globales se presentan como inaplazables. La cascada diaria de acontecimientos y de información acaba generando cierta resistencia de los hechos mismos a ser interpretados. En este contexto, la obsesión por definir y acotar un objeto-campo para la comunicación parece haberse intensificado con la convergencia tecnológica, la multiplicidad de soportes, la hiperconectividad digital.

Existen poderosas razones para una reflexión sobre el fenómeno de la comunicación más allá del debate, que proseguirá, en torno a su estatuto disciplinario. En la coyuntura actual de múltiples desafíos -crisis de la razón, de la cultura, de identidad, del modelo productivo (del capitalismo), los flujos migratorios, los integrismos en sus diferentes formas y otras variantes de la intolerancia, el acceso al conocimiento, la desigualdad social, los conflictos internacionales, el cambio climático- urge asumir responsabilidades frente al mundo y frente al futuro. La comunicación está llamada a algo más, y más importante, que la búsqueda de un campo propio. Justamente, porque debe de asumir como propios los nuevos contextos sociales, políticos y culturales, puesto que juega en ellos un papel esencial, generador, protagonista.

Es preciso seguir reflexionando qué espacio es el que corresponde al pensamiento comunicacional y qué puede aportar este como lugar estratégico a una época como la nuestra, a la intemperie, con enormes dificultades para pensarse a sí misma. Hemos de aspirar a un estatuto disciplinario para la comunicación que se proponga desafiar lo existente. Crear teoría para cuestionar lo real. Transformar, no contentarnos con describir o analizar. Que la teoría, en la línea propuesta por Feyerabend (1986), constituya un acontecimiento en el propio universo que describe. Este desafío no puede venir únicamente de una única perspectiva o una metodología sino que, por el contrario, ha de acometerse desde una metodología múltiple.

El ritmo del crecimiento demográfico, la aceleración de la historia en Hobsbawm (1998), la lentitud a la que alude Baudrillard para que los acontecimientos puedan condensarse y cristalizar en historia, la articulación de velocidad y política en Virilio (2006) o el arte de demorarse propuesto por Byun-Chul Han. Todo ello sugiere, por un lado, la posibilidad de plantear la idea de tempo como categoría epistemológica de las ciencias sociales. Por otro, en esta sociedad frenética, nos habla de la necesidad de encontrar un ritmo social, política y ambientalmente sostenible, de reivindicar la contemplación y el sosiego como contra hegemonía, como parte de una nueva agenda humanista. ¿Cuántas veces necesitamos 
escuchar al Ángel de la Historia de Benjamin para sentirnos vitalmente interpelados? ¿Volverá a florecer el desierto de Baudrillard? Reivindicar la dimensión ética y social de la comunicación -la comunicación como encuentro, como construcción de lugares comunes- se convierte hoy en un imperativo moral.

Notas

(1) Todas las traducciones incluidas en este artículo son nuestras.

(2) La literatura ofrece otras formas para el término Ciencias Sociales, como el de Ciencias del Hombre o, tal y como aparece en Merleau-Ponty (2011), Ciencias Humanas. En las fuentes analizadas, el término Ciencias Sociales es mayoritario entre los estudios en comunicación.

\section{Bibliografía}

Andacht, F. (2001). Un camino indisciplinario hacia la comunicación: medios masivos y semiótica. Bogotá: CEJA, UNESCO.

Ares Yebra, J. (2017). Pierre Souvtchinsky: desdoblamiento y concentración en Igor Stravinsky. (Tesis doctoral). Repositorio Institucional, Universidad de Vigo. Recuperado de http://www.investigo.biblioteca.uvigo.es/xmlui/handle/11093/866

Barberis, E. (2015). Teoría y metateoría de la comunicación: el desarrollo del campo. VIII Seminario Regional (Cono Sur). ALAIC, Políticas, actores y prácticas de la comunicación: encrucijadas de la investigación en América Latina.

Baudrillard, J. (1997). El otro por sí mismo (traducción de Joaquín Jordá). Barcelona: Anagrama.

Carey, J. W. (1992). Communication as Culture. Essays on Media and Society. New York: Routledge.

Cisneros, J. (2002). El concepto de la comunicación: el cristal con que se mira. Ámbitos, 7-8, 49-82.

Comité Invisible (2014). A nuestros amigos. Ciudad de México: Anarquía es una sinfonía.

Comité Invisible (2017). Ahora (traducción del francés de Diego Luis Sanromán). Logroño: Pepitas de calabaza.

Craig, R. T. (1999). Communication theory as a field. Communication Theory, 9, 119-161.

Donsbach, W. (2006). The identity of Communication Research. Journal of Communication, 56, 437-448. doi: 10.1111/j.1460-2466.2006.00294.x 
Feyerabend, P. (1986). En contra del método. Esquema de una teoría anarquista del conocimiento (traducción de Diego Ribes). Madrid: Taurus.

Galindo Cáceres, J. (2008). Filosofía y Comunicología. Exploración general para un programa posible de estudios. Razón y palabra, 13(64), 1-45.

Grossberg, L. (2012). Estudios culturales en tiempo futuro. Cómo es el trabajo intelectual que requiere el mundo de hoy (traducción de María Gabriela Ubaldini). Buenos Aires: Siglo XXI.

Habermas, J. (1999). Teoría de la acción comunicativa: racionalidad de la acción (Vol. I) (traducción de Manuel Jiménez Redondo). Madrid: Taurus.

Han, Byun-C. (2014). En el enjambre (traducción de Raúl Gabás). Barcelona: Herder.

Han, Byun-C. (2015). El aroma del tiempo. Un ensaño filosófico sobre el arte de demorarse (traducción de Paula Kuffer). Barcelona: Herder.

Handke, P. (1995). La tarde de un escritor (traducción de Isabel García-Wetzler). Madrid: Alfaguara.

Hobsbawm, E. (1998). Historia del siglo XX (traducción de Juan Faci, Jordi Arnaud y Carme Castells). Buenos Aires: Crítica.

Huizinga, J. (2001). Homo ludens (traducción de Eugenio Imaz). Madrid: Alianza.

James, W. (1994). Principios de psicología (traducción de Agustín Bárcena). México: Fondo de Cultura Económica.

Jarauta, F. (1991). Presentación. En La transformación de la conciencia moderna (pp. 7-13). Murcia: Universidad, Secretariado de Publicaciones.

Jaspers, K. (1964). Autobiografía filosófica (versión castellana de Pablo Simón). Buenos Aires: Sur.

Khanna, P. (2017). Conectografía. Mapear el futuro de la civilización mundial (traducción de Pablo Hermida Lazkano). Barcelona: Paidós.

Laclau, E. (2000). Nuevas reflexiones sobre la revolución de nuestro tiempo. Buenos Aires: Nueva Visión.

León Duarte, G. A. (2010). Estrategias, posiciones y prácticas científicas en la enseñanza y la investigación de la Comunicación en América Latina. Miguel Hernández Communication Journal, 1, 53-77.

Littlejohn, S. W. y Foss, K. A. (2005). Theories of human communication. Belmont, CA: Thomson-Wadsworth.

Lyotard, J-F. (1989). ¿Por qué filosofar? Cuatro conferencias. Barcelona: Paidós.

Lyotard, J-F. (1998). Lo inhumano. Charlas sobre el tiempo (traducción de Horacio Pons). Buenos Aires: Manantial. 
Martín-Barbero, J. (1984). De la Comunicación a la Cultura: perder el "objeto" para ganar el proceso. Signo y Pensamiento, 3(5), 17-24.

Martín-Barbero, J. (1992). Pensar la sociedad desde la comunicación. Un lugar estratégico para el debate de la modernidad. Diálogos de la comunicación, 1-8.

Martín-Barbero, J. (2007). Pensar la sociedad desde la comunicación. Revista Colombiana de Sociología, 29, 11-32. doi: 10.15446/rcs

Mattelart, A. y Mattelart, M. (1997). Historia de las teorías de la comunicación (traducción de Antonio López Ruiz y Fedra Egea). Barcelona: Paidós.

Mcluhan, M. (1964). Understanding Media. The Extensions of Man. New York: McGraw-Hill.

Merleau-Ponty, M. (2011). La fenomenología y las ciencias humanas (traducción de Raúl Carioli). Buenos Aires: Prometeo.

Pasquali, A. (1990). Comprender la comunicación. Venezuela: Monte Ávila Latinoamericana.

Pasternak, B. (1959). Poesías y otros escritos (Estudio preliminar y traducción de Vicente Gaos). Madrid: Guadarrama.

Paz, O. (2015). El laberinto de la soledad. Madrid: Cátedra.

Popper, K. (2008). La lógica de la investigación científica (traducción de Víctor Sánchez de Zavala). Madrid: Tecnos.

Rieznik, P. (2007). Las formas del trabajo y la historia. Una introducción al estudio de la economía política. Buenos Aires: Biblos.

Rizo García, M. (2012). Imaginarios sobre la comunicación. Algunas certezas y muchas incertidumbres en torno a los estudios de comunicación, hoy. Barcelona: Institut de la Comunicació, Universitat Autónoma de Barcelona.

Rizo García, M. (2014). El papel de las teorías de la comunicación en la construcción del campo académico de la comunicación. Reflexiones desde la historia, la epistemología y la pedagogía. Correspondencias \& Análisis, 4, 239-258.

Sandoval, L. R. (2015). Campo disciplinario de la comunicación: tensiones en su definición y enseñanza. Revcom, 16-27.

Sfez, L. (2007). La Comunicación. Buenos Aires: Amorrortu.

Souvtchinsky, P. (1982). Stravinsky auprès et au loin. Neuf paragraphes. En Lesure, F. (éd.). Stravinsky. Études et témoignages (15-52). París: Lattès.

Souvtchinsky, P. (1962). Le miracle du Sacre du Printemps (tradition et inspiration). Parcialmente inédito, mecanografiado en francés.

Vidales Gonzáles, C. (2017). De la comunicación como campo a la comunicación como concepto transdisciplinar: historia, teoría y objetos de conocimiento. Comunicación y Sociedad, 30, 45-68.

Question, Vol. 1, N.0 62, abril-junio 2019. ISSN 1669-6581

Instituto de Investigaciones en Comunicación | Facultad de Periodismo y Comunicación Social | Universidad Nacional de La Plata La Plata | Buenos Aires | Argentina

Página 17 de 18 
Virilio, P. (2006). Velocidad y política (traducción de Víctor Goldstein). Buenos Aires: la marca editora. 